\title{
Hand-movement profiles in a tactual-tactual matching task: Effects of spatial factors and laterality
}

\author{
JOËL FAGOT, AGNÈS LACREUSE, and JACQUES VAUCLAIR \\ Laboratory of Cognitive Neurosciences, Centre National de la Recherche Scientifique \\ Marseille, France
}

\begin{abstract}
We examined the effect of spatial factors and hemispheric lateralization upon hand-scanning strategies in 14 right-handed men tested in a tactual-tactual matching task. The experiment involved comparisons (judgments of same or different) between two objects sequentially touched by the fingertips of the left or right hand. Stimuli were made of smoothly joined cubes whose junctions were not haptically discernible. Exploratory strategies were inferred from the durations and locations of hand contacts with any of the cubes composing the stimuli. Accuracy was greater when the same stimulus was touched twice by the same hand than when different hands were used to feel it. With regard to strategies, both hands touched the upper parts of the object longer than the lower parts. Subjects also inspected more portions of the objects ipsilateral to the hand used. Overall differences in time spent touching cubes were greater for the right hand than for the left hand, showing that touch times were less evenly distributed on object parts for the former than for the latter. In this study, the process of information gathering by touch appears to be determined by the intertwining integration of contextual factors (e.g., stimulus position in space), biomechanical constraints on hand movements, and such cognitive factors as hemispheric differences on the ability to encode spatial pattern information.
\end{abstract}

The haptic perception system gathers information about the environment and the objects within it. This is an efficient system for information extraction, especially when familiar three-dimensional objects are felt (Klatzky, Lederman, \& Metzger, 1985). In haptic perception, the hands have both perceptual and motor functions. When a person feels an object by actively moving his/her hands, his/her perceptions depend on the stimulation of his/her cutaneous and kinesthetic receptors, which in turn depends on the shape of the stimulus and of course on the movements the person makes. The sensorial capacities of the haptic perceptual system are enhanced by the motor capacities of the hand, because the movements displace the sensorial receptors on the object and then expand the field of perception. As noted by Gibson (1962), active touch is not only a receptive sense, but also an exploratory one.

A number of investigators have shown that the contribution of exploratory movements to perception lies in some interactions between the nature of the stimulus and the nature of the scanning movements. For example, Goodnow, Baum, and Davidson (1971) showed that symmetrical curves were often felt as being skewed, but that

The authors thank Monique Chiambretto for writing the software, Bernard Arnaud for mechanical assistance, Raymond Fayolle for his aid with the electronics, and Victor Ginouvier for the preparation of Figure 1. Correspondence concerning this article should be addressed to J. Fagot, Cognitive Neuroscience Laboratory, CNRS, $31 \mathrm{ch}$. Joseph Aiguier, 13402, Marseille Cedex 20, France. the direction of the skew depended on the direction of the scanning. Locher and Simmons (1978) found that asymmetrical shapes were examined by smooth movements along the edge of the shape, whereas symmetrical forms were investigated by complete sweeps of the shape. Lederman and Klatzky (1987) noted that exploration procedures, defined as stereotyped movements, depended on the nature of the information to be extracted from the form. Hence, for instance, the hardness of a form would be assessed by applying torques or forces to parts of the object. In brief, the selection of exploratory hand movements and the resulting perception depend on multiple factors, including those related to stimulus attributes.

Among the possible factors that could influence exploratory strategies, one should emphasize hemispheric lateralization. There is compelling evidence that, at least in right-handed subjects, the left and right hemispheres differ in their abilities to process inputs from tactual, visual, and auditory senses (for a recent review, see Hellige, 1993). Hemispheric differences are especially apparent in the spatial domain. For example, the left hemisphere surpasses the right in categorizing a dot as being above or below a line. The right hemisphere is, in turn, better for evaluating the distance between two elements (Kosslyn et al., 1989). Others have proposed that the right hemisphere would treat the spatial information more holistically or globally than the left (for a review, see Bradshaw \& Nettleton, 1981). These hemispheric differences in spatial processing are expected to influ- 
ence haptic exploratory procedures, because each hand is primarily under the control of the contralateral hemisphere (Brinkman \& Kuypers, 1973).

Very little is known, however, about the lateralization of haptic exploratory procedures and their effects on perception. Nilsson and Geffen (1987) have shown, in a task implying a simultaneous perception of two objects by both hands, that performance was better when the hands moved in the same direction (e.g., clockwise) than when the two hands scanned the shapes in opposite directions. In our research group, we found that, during the investigation of composite shapes, the area inspected by the left hand was larger than that inspected by the right, both in monohaptic testing, that is, when only one hand was used at a time (Fagot, Lacreuse, \& Vauclair, 1993), and in dichhaptic testing, that is, when both hands are used simultaneously (Fagot, Hopkins, \& Vauclair, 1993). These findings on the lateralization of haptic exploratory procedures are congruent with the observation that performance in haptic form recognition can differ between the left and right hands (e.g., Flanery \& Balling, 1979; Verjat, 1988).

The present study is the third in a series of experiments in which we have attempted to specifically address the question of the lateralization of haptic exploratory procedures (Fagot, Hopkins, \& Vauclair, 1993; Fagot, Lacreuse, \& Vauclair, 1993). Previous reports on exploratory strategies have mainly focused on a description of the movements involved in haptic perception (e.g., Lederman \& Klatzky, 1987; Nilsson \& Geffen, 1987). The specificity of our approach resides in the analysis of hand contacts based on shape rather than hand movements per se. Complementary to the analyses concerning hand movements, this approach offers an insight into the way in which sensorial information is extracted. The present study has two original features with respect to our previous experiments. First, exploratory procedures are analyzed both when the subject has to memorize a stimulus and when he has to recognize it. Second, the analysis of manual strategies takes into account the shape of each stimulus in order to determine the effects of stimulus attributes on exploratory procedures.

\section{METHOD}

\section{Subjects}

Fourteen sighted men participated for pay in the experiment (mean age $=20.9$ years, $S D=3.1$ ). ${ }^{1}$ They reported themselves to be right-handed in each item of a 6-item laterality questionnaire (questions asked which hand was used in writing, drawing, ball throwing, teeth brushing, hammering, and using a racket), and indicated that they had no close relatives who were left-handed. They were not aware of the purpose of this study.

\section{Apparatus}

The apparatus was adapted from Fagot, Hopkins, and Vauclair (1993) and Fagot, Lacreuse, and Vauclair (1993). A technical description of that system is provided in Fagot, Arnaud, Chiambretto, and Fayolle (1992). Briefly, this apparatus consisted of an opaque $33 \times 33 \times 20 \mathrm{~cm}$ aluminum box inside of which two verti- cal tactile stimuli were concealed from view (see Figure 1). On the front of the box were two side-by-side vertically sliding doors operated by two motors. Raising the left or the right door provided a $7 \times 14.5 \mathrm{~cm}$ access to the stimuli. The rear inner side of the box was fitted with two adjacent plastic panels $(15.4 \times 16.4 \mathrm{~cm})$ on which the two vertical tactile stimuli were fixed $10 \mathrm{~cm}$ above the base of the box and $5 \mathrm{~cm}$ behind its front. ${ }^{2}$ A distance of $9 \mathrm{~cm}$ separated the inner sides of the two stimuli. The panels supporting the stimuli were mounted on springs above microswitches. Pushing a panel backward activated the microswitches fixed on its back side. The box was connected to an IBM-compatible PC computer via an $A / D$ converter.

The apparatus box lay on a $49.5 \times 20 \mathrm{~cm}$ plastic panel stuck onto a table. The experimenter could move the entire box $14.5 \mathrm{~cm}$ left or right by sliding it on the panel. When the box was at its extreme left position, the stimulus on its right was aligned with the midline axis of the subject. Similarly, the left stimulus was in line with the midline of the body when the box was shifted to the right side. A vertical $110 \times 65 \mathrm{~cm}$ carton board, with a central $8 \times 15 \mathrm{~cm}$ aperture on its base, was fixed in front of the box apparatus. The board prevented subjects from viewing the experimenter as well as components of the system other than the two doors.

\section{Stimuli}

Twelve tactile stimuli, comprising two identical sets of six different stimuli, were used (see Figure 2). The stimuli were threedimensional nonsense shapes made of several adjacent $1 \times 1 \times 1 \mathrm{~cm}$ metallic cubes fixed on a $6.5 \times 6.5 \mathrm{~cm}$ Perspex baseboard. Adjacent cubes were separated by a thin layer of plastic material in such a way as to electrically insulate all of them from each other. Use of similar stimuli in our previous studies had revealed that, when asked for a verbal description of the material after the experiment, subjects never reported that the objects were made up of cubes, but rather commented on their contours. Hence, one can consider that junctions between the cubes were not haptically discernible for normal subjects, at least in our testing conditions.

To equate the complexity of the stimuli, the six different tactile objects were all devised according to the following four rules: (1) They were all made up of eight cubes; (2) they were constructed with a maximum of five cubes in a row (this constraint

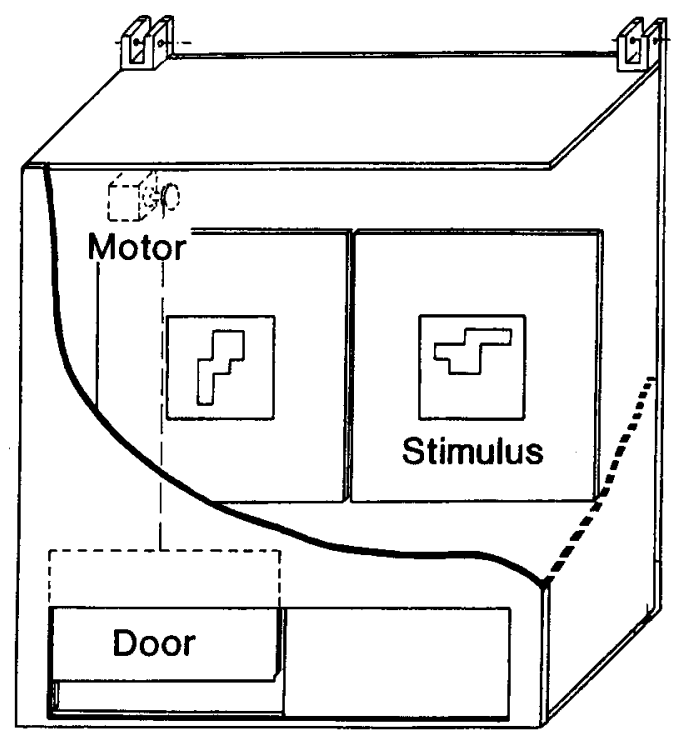

Figure 1. Schematic view of the apparatus, showing the two motorized front doors and the two tactile stimuli concealed from view within the box. 


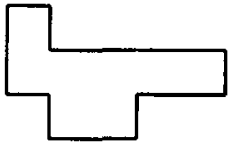

Form 1
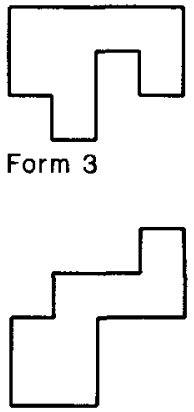

Form 5

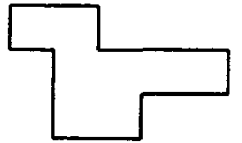

Form 2

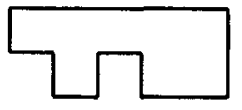

Form 4

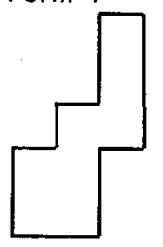

Form 6
Figure 2. Outlines of the six different stimuli. Forms depicted on the same line (e.g., Forms 1 and 2) constitute a pair of stimuli as they were presented in the experiment.

applied to height and width); (3) they were asymmetric with respect to both the horizontal and vertical planes; and (4) their contours comprised 10 angles and 10 sides.

\section{Procedure}

The experiment was run in a quiet room. The subject was seated at a table, facing the front side of the apparatus. At the onset of each trial, the experimenter placed two tactile stimuli within the apparatus box. One of these stimuli was used as the standard stimulus, that is, the stimulus to be recognized. The other stimulus served as the comparison stimulus. The box apparatus was shifted laterally in such a way that the standard stimulus was aligned with the midsagittal plane of the subject.

Each trial consisted of two consecutive phases. The first began with a brief warning tone, after which the subject was to adopt a symmetrical posture with his two hands on the table. One second after the onset of the signal, a red or green light-emitting diode (LED), fixed centrally above the apparatus on the carton board, was turned on. This signal indicated the hand (left or right) the subject was to employ to tactually inspect the standard stimulus. Three seconds after the warning tone, the door facing the standard stimulus was opened. The subject then introduced the designated hand into the box in order to explore the standard stimulus. The other hand remained on a grounded home panel $(60 \times 35 \mathrm{~cm})$ stuck on the table along the front side of the apparatus box. Because the subject was grounded by having one hand lying on the home panel, any hand contact with a cube composing the standard stimulus shifted its voltage from an initial value of $5 \mathrm{~V}$ to $0 \mathrm{~V}$. These electric variations were recorded on-line by the computer and later used to analyze haptic strategies. Overall, $10 \mathrm{sec}$ were allowed for exploration of the standard stimulus. Timing started when the hand touched one of the cubes composing the form. At the end of the 10 -sec period, a tone was delivered, and $1 \mathrm{sec}$ later the door was closed and the subject replaced both hands on the home panel. Then the experimenter slid the apparatus box laterally in order to position the comparison stimulus in the midsagittal plane of the subject, that is, in the exact same place as the standard stimulus in the first phase.

The second phase began when either a green or a red LED was turned on, depending on the hand to be used to touch the comparison form. Four seconds after the first door was closed, the door facing the comparison stimulus was opened. The subject intro- duced the designated hand into the apparatus and digitally examined the comparison form while the other hand remained on the grounded home panel. Hand contacts on cubes were recorded online using the same procedure as before. After having inspected the comparison form, the subject was requested to indicate whether the comparison stimulus was identical to or different from the standard stimulus. He gave his response by pushing the comparison stimulus backward, either once or twice, according to his same versus different judgment. This mode of response was selected because it did not imply verbal mediation. In effect, given the involvement of the left hemisphere in verbal processing, verbal mediation may potentially bias laterality effects on hand strategies. Seven subjects had to push the comparison form twice when the comparison stimulus matched the standard form. The other seven subjects had to push it once in the same case. Touch times were recorded from the first contact of the hand with any part of the comparison shape to the first push on the stimulus. There were no time constraints and there was no feedback regarding the subject's judgment of similitude between the standard and comparison forms.

On half of the trials for each subject, the comparison form was identical to the standard stimulus; on the other half, it was different from the standard. Additionally, four hand conditions were retained, depending on the hand (left or right) used to touch the standard stimulus and the one (left or right) employed to inspect the comparison stimulus. This design resulted in a total of 48 trials per subject, corresponding to 6 shape stimuli $\times 2$ pair types (same stimulus, different stimuli) $\times 4$ hand conditions (L-L, L-R, R-R, $\mathrm{R}-\mathrm{L})$. The order of trial presentations was selected randomly for each subject, and was therefore different for each subject.

Preliminary (unpublished) observations had shown that subjects made very few recognition errors when a standard stimulus was very different from some comparison stimuli (see also Gottheil, Cholewiak, \& Sherrick, 1978). Hence, we decided to maximize the resemblance between the standard and the comparison stimuli with the aim of getting a sufficient number of errors to perform error analyses. Three pairs of distinct stimuli were used in the trials in which the two stimuli to be felt were different. Form pairs are shown in Figure 2. Within a pair, each stimulus served equally frequently as the standard stimulus and as the comparison stimulus.

Although the instructions given to the subjects stressed speed in responding, they also asked the subjects to be as accurate as possible. In addition, the subjects were instructed to explore the stimuli tactually by using several fingers simultaneously. Prior to the test, the subjects received two practice trials with stimuli that were different from those used in the actual test.

\section{Dependent Variables}

On each trial, the computer recorded the location and duration of any hand contact with the cubes composing the standard and comparison forms. These data were later considered in order to derive hand-exploratory strategies. In practice, two temporal dependent variables were retained for data analysis. The first was the overall touch time, that being the overall duration of hand contacts with any part of the standard or comparison stimulus. The second was the cube touch time - that is, for each trial, the overall duration of hand contacts with each cube composing the standard and comparison shapes. Finally, accuracy scores were also recorded. Accuracy scores were defined as the total number of correct responses over the 48 trials performed by each subject.

\section{RESULTS}

\section{Accuracy Scores}

On average, subjects were correct on $34.2(S D=5.5)$ of the 48 test trials. Numbers of correct responses for each subject and condition are shown in Table 1. Accu- 
racy was not drastically affected by practice, as demonstrated by similar scores in the first 24 trials $(M=16.6)$ as compared with those of Trials $25-48(M=17.5)$. An analysis of variance (ANOVA) was performed on accuracy scores in order to determine if performance depended on the hand used. Independent variables for the ANOVA were the hand (left, right) used to scan the standard stimulus and the hand (left, right) used to scan the comparison stimulus. No significant main effect or interaction emerged. Note, however, that when the left hand examined the comparison stimulus, scores were slightly higher ( $M=17.7$ for 24 trials) than when the right hand was employed $[M=16.4 ; F(1,13)=4.06, p<.10]$.

A complementary ANOVA considered scores depending on pair type (same stimulus, different stimuli), and whether the same hand or different hands touched the standard and comparison stimuli. The sole significant effect was the pair type $\times$ hand combination interaction $[F(1,13)=10.95, p<.01]$. This interaction is depicted in Figure 3. Post hoc analyses (Tukey's honestly significant difference, $p<.05$ ) showed that when only one hand was used to feel the standard and the comparison stimuli, scores were greater in the same stimulus condition ( $M=9.2$ for 12 trials) than in the different stimulus condition $(M=7.4)$. Additionally, when two different stimuli were used during a trial, scores were greater in the different-hands condition $(M=9$ for 12 trials) than in the same-hand condition $(M=7.4)$.

\section{Overall Touch Times}

In an initial analysis of temporal variables, we sought to determine if overall touch times differed in correct and incorrect trials. Two ANOVAs were computed independently for the standard and comparison forms, with the outcome of the trial (success, failure) being the sole within-subject variable. The effect of outcome was not significant for the standard stimulus $(p>.10)$. For the comparison form, overall touch times were longer for incorrect $(M=7,029 \mathrm{msec})$ than for correct trials $[M=$ $5,976 \mathrm{msec} ; F(1,13)=30.6, p<.001]$. The shorter touch time for successful trials rules out a possible tradeoff between speed and accuracy.

Because of the above effect of outcome on overall touch times, additional analyses focused on correct trials only. Correct overall touch times were subjected to a three-way ANOVA with stimulus type (standard, comparison), hand (left, right), and identity of stimulus (1, $2,3,4,5,6)$ serving as the within-subject variables. This analysis showed a significant effect of stimulus type $[F(1,13)=21.7, p<.001]$, corresponding to longer touch times for the standard than for the comparison form (see mean data in Table 1). Other main effects or interactions were not significant. Finally, an ANOVA was computed to examine correct overall touch times for the comparison form depending on pair type (same, different) and whether or not the same or different hands felt the two stimuli. None of the main effects or interactions proved to be significant $(p>.1)$.

\section{Cube Touch Times}

We analyzed cube touch times for each stimulus independently. For each of the six stimuli, an ANOVA was performed on correct trials, with stimulus type (standard, comparison), hand (left, right), and cube identity

Table 1

Numbers of Correct Trials (CT) and Correct Overall Touch Times (COTT; in Milliseconds) Depending on Stimulus Type, Hand Used, and Pair Type

\begin{tabular}{|c|c|c|c|c|c|c|c|c|c|c|c|c|}
\hline \multirow[b]{5}{*}{ Subject } & & & & & \multicolumn{8}{|c|}{ Comparison Stimulus } \\
\hline & \multirow{2}{*}{\multicolumn{4}{|c|}{ Standard Stimulus }} & \multicolumn{4}{|c|}{ Left Hand } & \multicolumn{4}{|c|}{ Right Hand } \\
\hline & & & & & \multirow{2}{*}{\multicolumn{2}{|c|}{$\begin{array}{c}\text { Same } \\
\text { Stimuli } \dagger\end{array}$}} & \multirow{2}{*}{\multicolumn{2}{|c|}{$\begin{array}{l}\text { Different } \\
\text { Stimuli } \dagger\end{array}$}} & \multirow{2}{*}{\multicolumn{2}{|c|}{$\begin{array}{c}\text { Same } \\
\text { Stimuli } \dagger\end{array}$}} & \multirow{2}{*}{\multicolumn{2}{|c|}{$\begin{array}{l}\text { Different } \\
\text { Stimuli } \uparrow\end{array}$}} \\
\hline & \multicolumn{2}{|c|}{ Left Hand* } & \multicolumn{2}{|c|}{ Right Hand* } & & & & & & & & \\
\hline & $\mathrm{CT}$ & COTT & $\mathrm{CT}$ & COTT & CT & COTT & $\mathrm{CT}$ & COTT & $\mathrm{CT}$ & COTT & $\mathrm{CT}$ & COTT \\
\hline 1 & 20 & 8,293 & 22 & 8,256 & 11 & 6,144 & 12 & 4,383 & 11 & 5,823 & 8 & 4,732 \\
\hline 2 & 16 & 6,638 & 13 & 6,983 & 4 & 6,852 & 10 & 4,932 & 7 & 5,110 & 8 & 5,631 \\
\hline 3 & 16 & 6,511 & 16 & 5,186 & 8 & 5,627 & 8 & 8,442 & 11 & 5,528 & 5 & 9,794 \\
\hline 4 & 13 & 8,962 & 18 & 8,244 & 10 & 8,004 & 7 & 5,851 & 8 & 6,838 & 6 & 4,109 \\
\hline 5 & 20 & 7,866 & 21 & 7,700 & 9 & 6,459 & 11 & 6,714 & 11 & 7,191 & 10 & 4,963 \\
\hline 6 & 19 & 8,153 & 15 & 7,955 & 9 & 8,335 & 8 & 7,701 & 9 & 8,230 & 8 & 8,730 \\
\hline 7 & 15 & 6,834 & 14 & 7,213 & 9 & 5,825 & 6 & 6,323 & 7 & 7,440 & 7 & 5,248 \\
\hline 8 & 12 & 8,322 & 15 & 8,370 & 9 & 6,586 & 5 & 9,087 & 7 & 6,473 & 6 & 7,466 \\
\hline 9 & 15 & 5,591 & 16 & 5,716 & 9 & 3,759 & 10 & 3,094 & 4 & 3,319 & 8 & 3,394 \\
\hline 10 & 14 & 7,189 & 14 & 6,971 & 9 & 4,251 & 7 & 8,535 & 8 & 4,837 & 4 & 9,217 \\
\hline 11 & 20 & 7,941 & 17 & 7,695 & 8 & 5,650 & 12 & 6,764 & 8 & 4,774 & 9 & 6,091 \\
\hline 12 & 20 & 7,592 & 20 & 8,079 & 11 & 4,599 & 8 & 5,308 & 10 & 5,793 & 11 & 4,869 \\
\hline 13 & 22 & 8,629 & 21 & 8,485 & 11 & 4,491 & 9 & 4,160 & 10 & 4,746 & 12 & 4,598 \\
\hline 14 & 19 & 8,425 & 16 & 8,530 & 11 & 5,804 & 8 & 4,538 & 9 & 6,318 & 7 & 5,190 \\
\hline Mean & 17.2 & 7,639 & 17 & 7,527 & 9.1 & 5,885 & 8.6 & 6,130 & 8.6 & 5,887 & 7.8 & 6,002 \\
\hline$S D$ & 3.1 & 960 & 2.9 & 1,026 & 1.8 & 1,337 & 2.1 & 1,839 & 2 & 1,301 & 2.2 & 2,000 \\
\hline
\end{tabular}

Grand mean in overall touch time: standard $=7,583 \mathrm{msec}$, comparison $=5,976 \mathrm{msec}$, same $=5,886 \mathrm{msec}$, and different $=6,066 \mathrm{msec}$.

*24 test trials. $\$ 12$ test trials. 


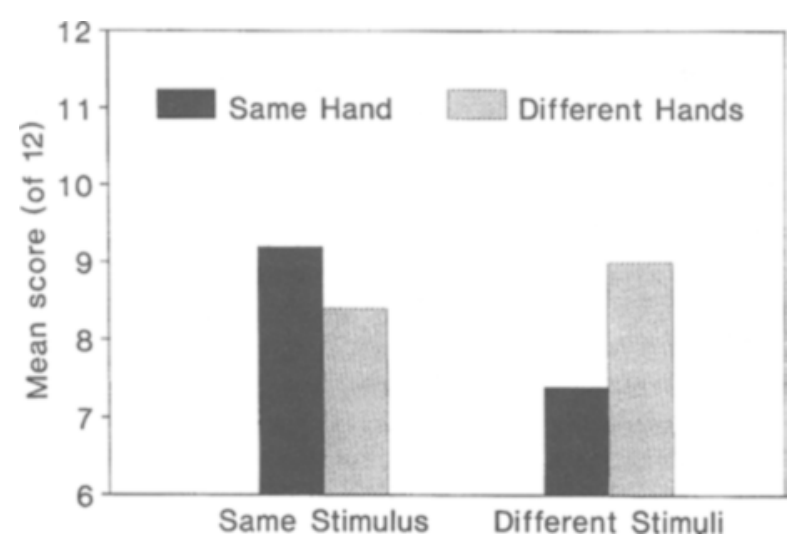

Figure 3. Scores depending on pair type (same, different stimuli) and whether or not the same hand was used to feel the standard and comparison stimuli.

( 8 cubes per stimulus) serving as the within-subject variables. Because pair type (same, different stimuli) did not affect overall touch times, this factor was discarded from the present analysis. Results of individual ANOVAs for each stimulus form are summarized in Table 2. The stimulus type had a reliable effect for each form. For all forms, this effect indicated longer exploration times for the standard than for the comparison form. The main effect of hand was never significant. The main effect of cube was strongly significant for each stimulus $(p<.001)$, showing that the cube touch times were not evenly distributed among the eight cubes that composed each stimulus. In terms of interaction, the most noticeable and consistent result comprised the hand $\times$ cube interactions, which were reliable for all stimuli $(p<.05)$.

A realistic representation of the hand $\times$ cube interactions is provided in Figure 4, which shows, for each hand and stimulus, the cube touch times ordered from 1 to 8 . In Figure 4, the lower numbers refer to the less often touched cubes; the higher numbers refer to the more often touched cubes. Figure 4 also indicates, on the basis of post hoc analyses (Tukey's honestly significant differences, $p<.05$ ), when significant intercube differences were found in cube touch times. Several conclusions can be drawn from Figure 4. First, subjects tended to touch more often the parts of the shape that were ipsilateral to the hand used. Thus, when the left hand was used, the left parts of the shape were more often touched, and vice versa. This effect is mostly true for stimuli numbered 1, 2, 3, and 4. Second, Figure 4 also shows a trend to touch the upper parts of the shapes more and, consequently, to touch the lower parts less. Again, this effect is most apparent for Form Pairs 1, 3, and 4. Finally, with reference to the differences between the left and right hands, post hoc analyses (Tukey's honestly significant difference, $p<.05$ ) showed more significant intercube differences for the right hand than for the left. This effect clearly emerges for Forms 2, 4, 5, and 6 (but see Form 1).
The latter result demonstrates that the right hand (left hemisphere) shares its touch time on the different parts of the stimuli less evenly than does the left hand (right hemisphere). To thoroughly analyze this effect, we computed for each trial the cube-touch-time difference- that is, the difference in touch time between the less and more often touched cubes. The data were then subjected

Table 2

Stimulus Type (Standard, Comparison) $\times$ Hand (Left, Right) $\times 3$ Cube (8 Cubes per Stimulus) ANOVA on the Cube Touch Times for Each Stimulus Form

\begin{tabular}{|c|c|c|c|}
\hline Form & $d f$ & $F$ & $p$ \\
\hline \multicolumn{4}{|c|}{ Stimulus Type (T) } \\
\hline 1 & 1,13 & 17.65 & $<.001$ \\
\hline 2 & 1,13 & 5.63 & $<.05$ \\
\hline 3 & 1,13 & 10.21 & $<.01$ \\
\hline 4 & 1,13 & 14.54 & $<.01$ \\
\hline 5 & 1,13 & 11.77 & $<.01$ \\
\hline 6 & 1,13 & 10.77 & $<.01$ \\
\hline \multicolumn{4}{|c|}{ Hand $(\mathrm{H})$} \\
\hline 1 & 1,13 & 0.03 & n.s. \\
\hline 2 & 1,13 & 0.06 & n.s. \\
\hline 3 & 1,13 & 0.68 & n.s. \\
\hline 4 & 1,13 & 0.01 & n.s. \\
\hline 5 & 1,13 & 0.15 & n.s. \\
\hline 6 & 1,13 & 0.59 & n.s. \\
\hline \multicolumn{4}{|c|}{ Cube (C) } \\
\hline 1 & 7,91 & 16.59 & $<.001$ \\
\hline 2 & 7,91 & 12.03 & $<.001$ \\
\hline 3 & 7,91 & 14.50 & $<.001$ \\
\hline 4 & 7,91 & 18.27 & $<.001$ \\
\hline 5 & 7,91 & 10.96 & $<.001$ \\
\hline 6 & 7,91 & 8.14 & $<.001$ \\
\hline \multicolumn{4}{|c|}{$\mathrm{T} \times \mathrm{H}$} \\
\hline 1 & 1,13 & 2.68 & n.s. \\
\hline 2 & 1,13 & 0.11 & n.s. \\
\hline 3 & 1,13 & 1.88 & n.s. \\
\hline 4 & 1,13 & .02 & n.s. \\
\hline 5 & 1,13 & 3.24 & $<.10$ \\
\hline 6 & 1,13 & 0.02 & n.s. \\
\hline \multicolumn{4}{|c|}{$\mathrm{T} \times \mathrm{C}$} \\
\hline 1 & 7,91 & 3.43 & $<.01$ \\
\hline 2 & 7,91 & 2.56 & $<.02$ \\
\hline 3 & 7,91 & 0.18 & n.s. \\
\hline 4 & 7,91 & 1.71 & n.s. \\
\hline 5 & 7,91 & 1.80 & $<.10$ \\
\hline 6 & 7,91 & 1.00 & n.s. \\
\hline \multicolumn{4}{|c|}{$\mathrm{C} \times \mathrm{H}$} \\
\hline 1 & 7,91 & 21.07 & $<.001$ \\
\hline 2 & 7,91 & 25.43 & $<.001$ \\
\hline 3 & 7,91 & 7.10 & $<.001$ \\
\hline 4 & 7,91 & 7.16 & $<.001$ \\
\hline 5 & 7,91 & 4.70 & $<.001$ \\
\hline 6 & 7,91 & 2.49 & $<.05$ \\
\hline \multicolumn{4}{|c|}{$\mathrm{T} \times \mathrm{H} \times \mathrm{C}$} \\
\hline 1 & 7,91 & 0.93 & n.s. \\
\hline 2 & 7,91 & 1.05 & n.s. \\
\hline 3 & 7,91 & 2.66 & $<.02$ \\
\hline 4 & 7,91 & 4.36 & $<.001$ \\
\hline 5 & 7,91 & 3.39 & $<.01$ \\
\hline 6 & 7,91 & 1.31 & n.s. \\
\hline
\end{tabular}



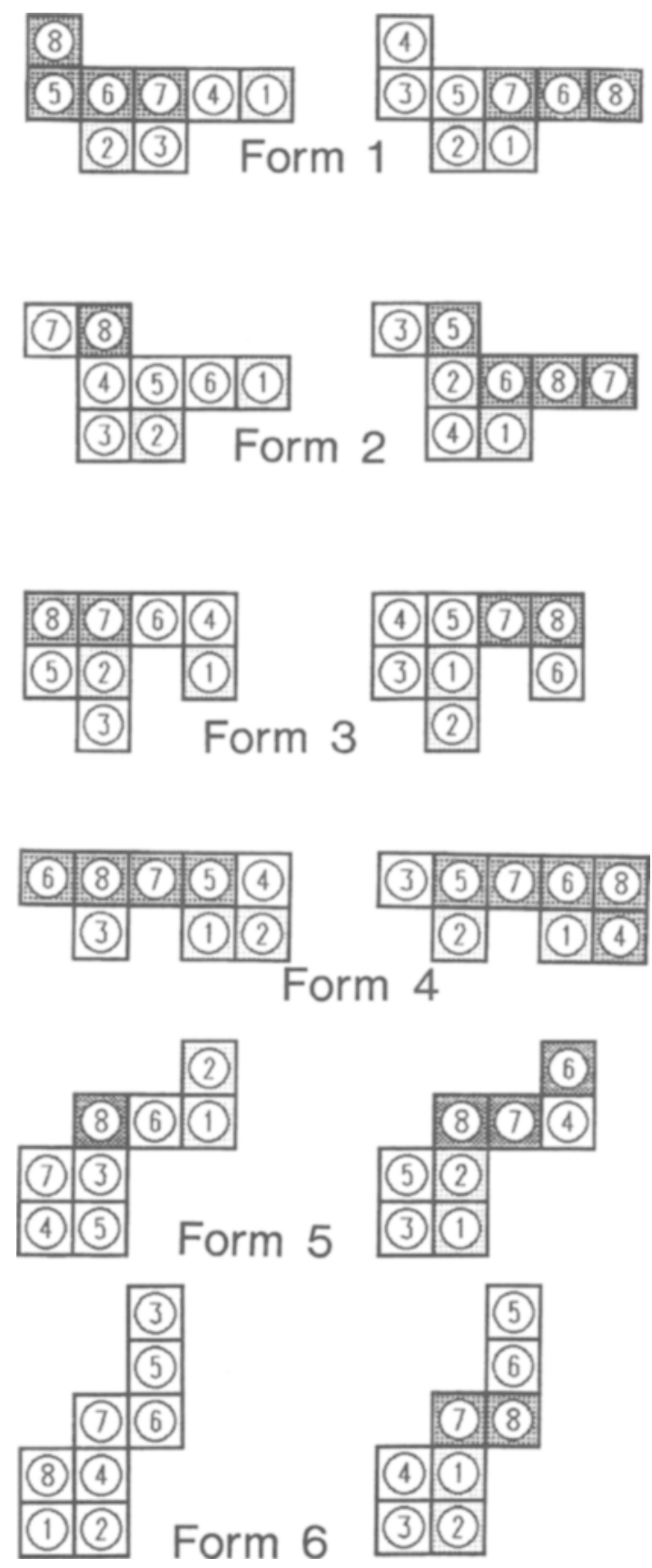

Figure 4. Distribution of the cube touch times for each stimulus $\times$ hand condition. Each line depicts the pattern of cube touch times when the same stimulus was investigated by the left hand (left side of the figure) and by the right hand (right side of the figure). Numbers indicated on the cubes show the ordered distribution of the cube touch times, with the lower times indicated by lower digits. Cube touch times for the shaded cubes were all significantly higher (Tukey's honestly significant difference, $p<.05$ ) than the cube touch times for dotted cubes. to a stimulus form (Forms 1-6) $\times$ stimulus type (standard, comparison) $\times$ hand (left, right) ANOVA. For reasons of conciseness, we will comment only on significant main effects and on the significant interactions of the highest order.

The main effect of stimulus type was significant $[F(1,13)=30.40, p<.001]$, corresponding to greater cube-touch-time differences for the standard than for the comparison stimulus. Also significant was the main effect of hand $[F(1,13)=6.66, p<.05]$. Cube-touch-time differences were greater for the right hand $(M=$ $3,466 \mathrm{msec})$ than for the left $(M=3,082 \mathrm{msec})$. Finally, the third-order stimulus form $\times$ hand $\times$ stimulus type interaction had a reliable effect $[F(5,65)=2.65, p<$ $.05]$. Results for this interaction are depicted in Table 3 . On average, hand differences were greater for the standard than for the comparison stimulus (note that the hand $X$ stimulus type interaction approached significance, $p=.08$ ), although this relationship was not constant for every tactual object (e.g., Form 4).

\section{DISCUSSION}

The purpose of this experiment was to examine hand movements in a tactual-tactual form-matching task. The results suggest that the scanning strategies are codetermined by a series of interrelated factors. In this discussion, we will first comment on the spatial factors of the task and their interaction with biomechanical constraints on hand movements. Then we will consider more cognitive factors, with an emphasis on the importance of hemispheric differences in the encoding of spatial information.

\section{Effects of Spatial Factors on Movement Profiles}

Our tactile apparatus was initially designed to enhance distal movements of the fingers in order to assess hemispheric differences in exploratory procedures. The spatial properties of tactile stimuli-namely, their fixity,

Table 3

Mean Cube-Touch-Time Differences (in Milliseconds) for Each Stimulus Form (6 Stimuli) $\times$ Stimulus Type (Standard, Comparison) $\times$ Hand (Left, Right) Condition

\begin{tabular}{lcrrrr}
\hline & \multicolumn{2}{c}{ Standard Stimulus } & & \multicolumn{2}{c}{ Comparison Stimulus } \\
\cline { 2 - 3 } \cline { 6 - 6 } Form & LH & RH & & LH & RH \\
\hline 1 & 3,272 & 4,273 & & 2,398 & 2,929 \\
2 & 3,143 & 3,867 & & 3,073 & 2,596 \\
3 & 3,576 & 4,112 & 3,043 & 3,271 \\
4 & 3,505 & 3,246 & 2,542 & 3,112 \\
5 & 3,625 & 4,363 & & 2,777 & 3,106 \\
6 & 2,901 & 3,926 & & 3,133 & 2,790 \\
Mean & 3,337 & 3,965 & & 2,828 & 2,967 \\
Grand Mean & \multicolumn{2}{c}{3,651} & & \multicolumn{2}{c}{2,897} \\
\hline
\end{tabular}

Note- - LH $=$ left hand, RH = right hand. Cube-touch-time differences correspond to the differences in the time spent on the less and more often touched cubes. 
their vertical position, and their confinement within a small-sized box - defined constraints limiting the number and type of exploratory movements that could be applied to the form. In practice, subjects investigated the forms by placing the hand upright, the thumb being located in the lower region of the shape and the other fingers being flexed toward the shape's upper part. From this "basic" position, information was gathered using a combination of lateral displacements of the thumb and up-down movements of the other fingers, with lateral and torque movements of the hand whose origin was the wrist. Lateral and torque movements allowed for the exploration of the whole stimulus shape by the thumb and the index finger.

We believe that this movement profile could explain at least three of our results. First, the upper portions of the stimulus were touched for a longer time than the lower portions (see Figure 4). This effect could derive from the location of the fingers whose fields of action were, due to hand posture and, except for the thumb, mostly located on the top of the shape.

Also explainable by the movement profile is the finding that regions ipsilateral to the hand used were touched more than contralateral regions (see Figure 4). This second result might reflect motions of the hands along the left-right axis. When the right hand was employed, the right part of the shape could be perceived by every finger, including the ulnar ones. By contrast, the left part of the shape was principally touched by the indexthumb grip, because these fingers always remained in contact with the stimulus. This effect was left-right reversed when the left hand was used.

The third result that could be explained by the movement profile is the finding that accuracy scores were greater for same pair types than for different pair types, when only one hand was used to feel both the standard and comparison stimuli. Given that each hand tended to focus on ipsilateral parts of the objects, when only one hand was used, the locus of exploration is likely to have been identical for both stimuli. This bias could lead subjects to detect the similarities between the stimuli rather than their differences, leading thus to greater accuracy scores for the same pair type.

Although the aforementioned effects were due to the hardware used and to the biomechanical constraints of the movements, they provide a frame of reference on which cognitive factors can operate.

\section{Effects of Cognitive Factors on Movement Profiles}

In the present study, some important differences in the scanning procedures exhibited by the left and right hands were found. Cube touch times were less equally distributed on stimulus parts for the right hand than for the left (Figure 4). Cube-touch-time differences were also greater for the right hand than for the left. Because digital movements are controlled by the hemisphere opposite to the hand used (Brinkman \& Kuypers, 1973), these data indicate that the two cerebral hemispheres have adopted distinct processes of information gathering.
An important literature is available on the respective abilities of each hemisphere to process tactual information (for a recent review, see Summers \& Lederman, 1990). This literature often reports a left-hand advantage (right hemisphere) in the recognition of meaningless shapes (but see Yandell \& Elias, 1983). A left-hand advantage was found, for instance, by Benton, Levin, and Varney (1973) in the tactile perception of orientations. Verjat (1988) found a superiority of the left hand in the recognition of nonmeaningful stimuli. Likewise, Fagot, Hopkins, and Vauclair (1993) found, in a dichhaptic task, that visual recognition of a tactile form was greater when the shape was initially touched by the left hand than when it was initially touched by the right. Such lefthand advantages in tactual recognition were said to reflect a right-hemisphere advantage for the treatment of spatial information (e.g., Flanery \& Balling, 1979; Verjat, 1988), at least when the task did not imply verbal mediation.

Besides a fairly extensive and diverse literature on hand differences in recognition scores, little is known so far on the lateralization of haptic scanning procedures. Nilsson and Geffen (1987) have shown that, in a dichhaptic task, recognition was better when two tactile shapes were explored in congruent (e.g., clockwise) directions than when the two hands scanned the shapes in different directions (i.e., one hand moving clockwise, the other moving counterclockwise). Using the same tactile apparatus as that used in the present study, Fagot, Lacreuse, and Vauclair (1993) found, in a monohaptic task, that the left hand (right hemisphere) simultaneously touched larger surface areas during stimulus scanning than did the right hand. ${ }^{3}$ This effect was also reported in a study (Fagot, Hopkins, \& Vauclair, 1993) that investigated the synchronization of hand movements when both hands had to feel and compare two objects simultaneously (i.e., in a dichhaptic task).

In the reports of our research group, hand differences were interpreted as suggesting a more global analysis of the stimuli for the left hand (right hemisphere) than for the right hand (left hemisphere). The current findings are in agreement with this interpretation. Cube-touch-time differences between hands suggest that the right hand (left hemisphere) would focus more on specific parts of the stimuli than would the left hand (right hemisphere). Under this hypothesis, the right hand would recognize the shape on the basis of specific features, whereas the left (right hemisphere) would recognize it more from its general structure. However, it should be kept in mind that attentional and memory factors are crucial for information extraction (see, e.g., Kinsbourne, 1970). Moreover, our apparatus tells us about which parts of the shape were touched, but all tactile sensorial information was not necessarily processed to encode the stimulus shape. Hence, this study calls for additional experiments in which one should try to relate patterns of hand scanning to the subject's knowledge of stimulus properties. It is nevertheless clear from the current study that lateralization 
is a critical factor in determining the way tactile objects are explored.

In the present study, the standard was touched longer than the comparison form was. It is possible that this difference derived from a procedural feature of the study, namely the differences in time constraints to explore the standard (i.e., a maximum of $10 \mathrm{sec}$ ) and comparison stimuli (i.e., emphasis on speed). But it is also possible that these time differences were due to more cognitive factors. Thus, when touching the standard, the subject selected components of stimulation which specified the shape, with a focus on its contour, which was the discriminative factor. When touching the comparison forms, the subject had to map information in memory with the features of the explored stimulus. His judgment of similarity between the two forms may have depended on the points of similarity or overlap in the properties he attributed to the two stimuli (e.g., Goodnow, 1971). Hence, the process of information extraction did not need to be as exhaustive for the comparison stimulus as it was for the standard stimulus, which is in agreement with the results obtained for touch time.

Complementary to the above effects, the effect of phase for cube-touch-time differences interacted with the hand used. Specifically, these hand differences were greater for the standard than for the comparison stimuli. We propose that the memorized representation of the standard stimulus and the need to recognize some of its features homogenizes the process of information gathering for each hand. If this reasoning is correct, then the subject's knowledge about the shape and his search for some typical attributes would be an additional factor that would influence exploratory procedures and their lateralization. This view is in agreement with Lederman and Klatzky's (1987) findings in showing that the selection of a given exploratory procedure (e.g., contour following) depends on the information to be extracted from the object (e.g., to pick up information about the contour).

It should finally be noted that the current study revealed neither accuracy score nor time differences between pair types (same, different). This result contrasts with the frequently reported finding that, under visual control, judgments are faster for same pair types than for different pair types (e.g., Krueger, 1978). In agreement with our findings, however, no such effect was found by Millar (1977) in a tactual matching task. The consistency in results between Millar's research and the current study suggests differences in processing pair types in the visual and the tactual modalities.

The present research has highlighted several stimulusand subject-related factors that could influence or even determine haptic exploratory procedures. It thus calls for a thorough investigation of the psychophysics of the form along with an investigation of exploratory strategies and accuracy scores to tap the essential features of active touch. In general terms, we think that a detailed analysis of hand contacts on the forms constitutes a heuristic approach toward obtaining a better under- standing of the somatosensory information and the cognitive strategies that subjects use to encode haptic spatial forms. Such an approach constitutes a useful complement to more global analyses of hand movements per se.

\section{REFERENCES}

Benton, A. L., Levin, H. S., \& Varney, N. R. (1973). Tactile perception of direction in normal subjects. Neurology, 23, 1248-1250.

Bradshaw, J. L., \& Nettleton, N. C. (1981). The nature of hemispheric specialization in man. Behavioral \& Brain Sciences, 4, 51 91.

Brinkman, C., \& KuYpers, H. G. J. M. (1973). Cerebral control of contralateral and ipsilateral arm, hand and finger movements in split brain rhesus monkeys. Brain, 96, 653-674.

Fagot, J., Arnaud, B., Chiambretto, M., \& Fayolle, R. (1992). Lateralization in haptic processing: An apparatus for analyzing manual strategies. Behavior Research Methods, Instruments, \& Computers, 24, 54-59.

FAGOT, J., Hopkins, W. D., \& VAuClair, J. (1993). Hand movements and hemispheric specialization in dichhaptic explorations. Perception, 22, 847-853.

Fagot, J., Lacreuse, A., \& Vauclair, J. (1993). Haptic discrimination of nonsense shapes: Hand exploratory strategies but not accuracy reveal laterality effects. Brain \& Cognition, 21, 212-225.

FlaneRY, R. C., \& Balling, J. D. (1979). Developmental changes in hemispheric specialization for tactile spatial ability. Developmental Psychology, 55, 291-297.

GiBson, J. J. (1962). Observations on active touch. Psychological Review, 69, 477-491.

Goodnow, J. J. (1971). Eye and hand: Differential memory and its effects on matching. Neuropsychologia, 9, 89-95.

Goodnow, J. J., Baum, B., \& Davidson, P. (1971). A haptic error: Skew in a symmetrical curve. Perception \& Psychophysics, 10, 253256.

Gottheil, E. F., Cholewiak, R. W., \& Sherrick, C. E. (1978). The discrimination of vibratory patterns on a tactile matrix. Bulletin of the Psychonomic Society, 11, 21-24.

Hellige, J. B. (1993). Hemispheric asymmetry: What's right and what's left. Cambridge, MA: Harvard University Press.

KinsBourne, M. (1970). The cerebral basis of lateral asymmetries in attention. Acta Psychologica, 33, 193-201.

Klatzky, R., Lederman, S. J., \& Metzger, V. A. (1985). Identifying objects by touch: An "expert system." Perception \& Psychophysics, 37, 299-302.

Kosslyn, S. M., Koening, O., Barrett, A., Cave, C. B., Tang, J., \& GABrIELI, J. D. E. (1989). Evidence for two types of spatial representations: Hemispheric specialization for categorical and coordinate relations. Journal of Experimental Psychology: Human Perception \& Performance, 15, 723-735.

Krueger, L. E. (1978). A theory of perceptual matching. Psychological Review, 85, 278-304.

LEDERMAN, S. J., \& KLATZKY, R. (1987). Hand movements: A window into haptic object recognition. Cognitive Psychology, 19, 342368.

LOCHER, P. J., \& Simmons, R. W. (1978). Influence of stimulus symmetry and complexity upon haptic scanning strategies during detection, learning, and recognition tasks. Perception \& Psychophysics, 23, 110-116.

MCGLONE, J. (1980). Sex differences in human brain asymmetry: A critical survey. Behavioral \& Brain Sciences, 3, 215-243.

Millar, S. (1977). Early stages of tactual matching. Perception, 6, 333-343.

Nilsson, J., \& Geffen, G. (1987). Perception of similarity and laterality effects in tactile shape recognition. Cortex, 23, 599-614.

Summers, D. C., \& Lederman, S. J. (1990). Perceptual asymmetries in the somatosensory system: A dichhaptic experiment and critical review of the literature from 1929 to 1986 . Cortex, 26, 201-226. VERJAT, I. (1988). Dissymmetry in manual perception by children on 
form recognition tasks with tactually presented forms. European Bulletin of Cognitive Psychology, 8, 223-239.

YANDELL, L., \& EliAS, J. W. (1983). Left hemispheric advantage for a visuospatial-dichaptic matching task. Cortex, 19, 69-77.

\section{NOTES}

1. We restricted the sample to male subjects because males are reported to be more lateralized than females (e.g., Fagot, Lacreuse, \& Vauclair, 1993; McGlone, 1980). This choice was thus expected to reveal subtle effects that might otherwise, say with a more heterogeneous subject sample, have been missed.
2. This system was originally built because of our interests in comparative studies involving humans and monkeys (see Fagot et al., 1992). In that context, the vertical presentation of the stimuli was imposed by the need to prevent monkeys from seeing the tactile stimuli regardless of their posture.

3. Although not reported in this paper for reasons of conciseness, this effect was again present in the data set $(p<.05)$.

(Manuscript received November 15, 1993; revision accepted for publication February 24, 1994.) 